\title{
Unraveling the structural basis for the exceptional stability of RNA G-quadruplexes capped by a uridine tetrad at the $3^{\prime}$ terminus
}

\author{
WITOLD ANDRAKOJĆ, ${ }^{1}$ MAGDALENA MAKGOWSKA, ${ }^{1}$ JOANNA SARZYŃSKA, KAROL PASTERNAK, \\ KAMIL SZPOTKOWSKI, RYSZARD KIERZEK, and ZOFIA GDANIEC
}

Institute of Bioorganic Chemistry, Polish Academy of Sciences, 61-704 Poznan, Poland

\begin{abstract}
Uridine tetrads (U-tetrads) are a structural element encountered in RNA G-quadruplexes, for example, in the structures formed by the biologically relevant human telomeric repeat RNA. For these molecules, an unexpectedly strong stabilizing influence of a U-tetrad forming at the $3^{\prime}$ terminus of a quadruplex was reported. Here we present the high-resolution solution NMR structure of the r(UGGUGGU) 4 quadruplex which, in our opinion, provides an explanation for this stabilization. Our structure features a distinctive, abrupt chain reversal just prior to the $3^{\prime}$ uridine tetrad. Similar "reversed U-tetrads" were already observed in the crystalline phase. However, our NMR structure coupled with extensive explicit solvent molecular dynamics (MD) simulations identifies some key features of this motif that up to now remained overlooked. These include the presence of an exceptionally stable $2^{\prime} \mathrm{OH}$ to phosphate hydrogen bond, as well as the formation of an additional $\mathrm{K}^{+}$binding pocket in the quadruplex groove.
\end{abstract}

Keywords: G-quadruplex; NMR spectroscopy; U-tetrad; molecular dynamics; potassium binding pocket

\section{INTRODUCTION}

G-quadruplexes constitute by far the most studied family of noncanonical nucleic acid structures. Historically, RNA Gquadruplexes received significantly less attention than their DNA counterparts (Joachimi et al. 2009). This situation is rapidly changing in the last few years, following the increasing amount of evidence that RNA quadruplexes play important roles in vivo (Millevoi et al. 2012; Biffi et al. 2014). They were shown to be implicated among others in translational regulation (Song et al. 2016), alternative splicing (Didiot et al. 2008; Marcel et al. 2011), and cellular stress response (Lyons et al. 2017). Another example are the G-quadruplexes formed by the varying length transcripts of the human telomeric sequence-the TERRA RNA-(Azzalin et al. 2007; Schoeftner and Blasco 2007) which are involved in the formation of telomeric heterochromatin and the regulation of the telomere length (Schoeftner and Blasco 2009). Structurally, RNA G-quadruplexes display much less diversity compared to their DNA equivalents as only the parallel quadruplex topology was observed in RNA so far (Malgowska et al. 2016). However,

\footnotetext{
1Joint first authors.

Corresponding author: zgdan@ibch.poznan.pl

Article is online at http://www.rnajournal.org/cgi/doi/10.1261/rna. 068163.118.
}

RNA G-quadruplexes are inherently more thermodynamically stable (Mergny et al. 2005; Arora and Maiti 2009), an effect whose origin is not yet fully understood (Zhou et al. 2017). Evidence gathered to date suggests that it is a combined effect of both the presence of $2^{\prime}-\mathrm{OH}$ group and of the substitution of thymine for uridine in RNA. The $2^{\prime}$-hydroxyl groups stabilize RNA G-quadruplexes by forming additional transient hydrogen bonds, both intramolecular and involving the surrounding solvent molecules (Agarwala et al. 2015). The substitution of thymidine for uridine on the other hand, diminishes hydrophobic interactions in quadruplex loops (Olsen and Marky 2009).

Both the uridine and thymidine residues can also exert a direct stabilizing effect on a G-quadruplex structure, through the formation of base quartets of their own: the U- (Cheong and Moore 1992) and T-tetrads (Patel and Hosur 1999), respectively. However, once again the presence of uridine is expected to be more favorable, as the T-tetrads suffer from an increased interbase steric repulsion (mostly between the methyl groups and $\mathrm{O} 4$ atoms) (Gu and

(C) 2019 Andrałojć et al. This article is distributed exclusively by the RNA Society for the first 12 months after the full-issue publication date (see http://rnajournal.cshlp.org/site/misc/terms.xhtml). After 12 months, it is available under a Creative Commons License (Attribution-NonCommercial 4.0 International), as described at http:// creativecommons.org/licenses/by-nc/4.0/. 
Leszczynski 2001). So far, uridine and thymidine residues capable of tetrad formation were most often observed on the $5^{\prime}$ or $3^{\prime}$ termini of tetramolecular quadruplexes. An analysis of the deposited crystal structures supports the greater propensity of uridines for tetrad formation. For example, the $3^{\prime}$-terminal thymidine residues appear to populate a wide range of conformations in the crystalline state. Apart from T-tetrads, the reported structures contain many instances of unpaired or disordered thymidines (Laughlan et al. 1994; Phillips et al. 1997; Cáceres et al. 2004; Creze et al. 2007; Lee et al. 2007). In contrast, for the $3^{\prime}$ uridines one observes a marked dominance of uridine tetrad (U-tetrad) forming conformers, yet still with some structural diversity present (Deng et al. 2001; Pan et al. 2003a,b,c, 2006a,b; Fyfe et al. 2015). The dominant conformation in the crystalline state featured a chain reversal just prior to the $3^{\prime}$-uridine residue, leading to the U-tetrad stacking with the G-stem with the opposite face and having its hydrogen bond directionality inverted with respect to the preceding G-tetrads. However, some crystals featured two other modes of $3^{\prime}$ U-tetrad formation, one with standard helical backbone conformation and yet another conformation with chain reversal, as summarized in Fyfe et al. (2015). In some crystals, two different conformations were found coexisting in the unit cell (Deng et al. 2001; Pan et al. 2003a; Fyfe et al. 2015). Thus, the structural data available to date suggest that 3 '-terminal U-tetrads, even though energetically favorable, exhibit noticeable structural heterogeneity.

Quite surprisingly, the experimentally observed stabilizing effect related to 3 -terminal U-tetrads turns out to be much greater than the structural data would suggest. The most striking illustration of this effect is the result reported by Xu et al. (2010). The authors have investigated two tetramolecular quadruplexes formed by $5^{\prime}$-UUAGGG-3' and 5'UAGGGU-3' molecules, both corresponding to a single repeat of the human telomeric sequence UUAGGG, yet taken in two different "reading frames." Most uridine residues did not assume well-defined conformations, with the exception of the $3^{\prime}$-uridine of $5^{\prime}$-UAGGGU-3' which formed a U-tetrad. Unexpectedly, these two quadruplexes have demonstrated a very pronounced difference in thermal stability, with the quadruplex capped by the 3 '-terminal U-tetrad displaying $29^{\circ} \mathrm{C}$ higher melting temperature. Recently, a similar difference in the melting temperature was observed between the quadruplexes $d(T) r(G G G) d(T)$ and $r(U G G G U)$, differing only by the replacement of $d(T)$ by $r(U)$ in the terminal residues (Zhou et al. 2017). The surprisingly high thermal stability of the $3^{\prime} U$-tetrad itself was already noticed in the very first paper reporting the experimental observation of U-tetrad formation. In that NMR study of the (UGGGGU) 4 quadruplex the imino protons of the $3^{\prime}$-terminal uridine could be observed even at $70^{\circ} \mathrm{C}$, advocating for the preservation of the U-tetrad at such an elevated temperature (Cheong and Moore 1992). To cite another example, a recently reported extremely stable RNA octaplex is also capped by a $3^{\prime}$-terminal U-tetrad (Xiao et al. 2017) whose presence may be one of the factors responsible for the overall stability.

Here we present a combined high-resolution nuclear magnetic resonance (NMR) and molecular dynamics (MD) study of the quadruplex formed by the r(UGGUGGU) molecule. Our previous NMR and mass spectrometry (MS) analysis of this quadruplex has allowed us to propose its overall topology (Malgowska et al. 2014). That study has revealed that r(UGGUGGU) adapts a tetramolecular, parallel-stranded structure, both in the presence of $\mathrm{K}^{+}$and $\mathrm{Na}^{+}$ ions. Furthermore, we demonstrated that all three uridine residues are involved in the formation of U-tetrads. This quadruplex was also shown to be extremely stable in $\mathrm{K}^{+}$-containing solution, conserving its fold even above $90^{\circ} \mathrm{C}$. The current study presents the high-resolution NMR structure, as well as unrestrained MD simulations of the r(UGGUGGU) 4 quadruplex. Our results reveal the presence of a well-defined conformation of the 3 '-terminal $U$ tetrad, featuring an abrupt chain reversal just prior to the uridine residue, similar to the state most often found in the crystal structures. Here, for the first time, we report such oligonucleotide chain reversal at the $3^{\prime}$-terminal uridine of a G-quadruplex in solution and ascertain that, at least in the case of the $r(U G G U G G U)_{4}$ quadruplex, it exists as a very rigid structural element, not involved in any conformational exchange processes. Moreover, we identify a series of, so far overlooked, unique structural properties of such a motif, that in our opinion constitute the structural basis for the exceptional thermal stability of RNA G-quadruplexes containing it. These features include remarkably stable sugar-phosphate hydrogen bonding anchoring U7 and $\mathrm{G6}$, as well as, the formation of an unexpected metal binding pocket in the quadruplex groove.

Furthermore, the structure presented here contains the first example of a U-tetrad stacked between two G-tetrads. In all the previously studied sequences, uridine residues in such context were bulged out (Pan et al. 2003c, 2006a). In addition, we also observe a 5 '-terminal U-tetrad, although it appears less stable than the other tetrads.

\section{RESULTS}

\section{NMR spectral features and assignment}

The NMR spectra of $r(U G G U G G U)_{4}$ contained a single set of resonances, which together with the information from MS (Malgowska et al. 2014), signifies that we are dealing with a parallel-stranded quadruplex with a fourfold symmetry axis (see the reasoning in Cheong and Moore 1992).

The NMR spectra were assigned using standard procedures for quadruplexes (Varani et al. 1996; Wijmenga and van Buuren 1998), as described in Malgowska et al. (2014) and in Materials and Methods. Several resonances 
belonging to the $\mathrm{G} 3$ and $\mathrm{U} 4$ residues were considerably broadened by exchange at room temperature. Their assignment in $\mathrm{K}^{+}$-containing conditions was aided by concurrent analysis of the spectra recorded at $55^{\circ} \mathrm{C}$, at which temperature these resonances yielded much sharper lines. All seven imino protons present in the molecule are clearly identifiable in the spectra recorded at low temperatures (Fig. 1), and thus involved in the formation of G- and U-tetrads. The imino proton resonances were assigned through combined analysis of the ${ }^{1} \mathrm{H}-{ }^{15} \mathrm{~N} \mathrm{HSOC}$ and NOESY spectra, as detailed in Malgowska et al. (2014). Each tetrad turned out to be composed of four symmetry-related copies of the same residue and from now on the tetrads will be referred to using residue numbers for simplicity. Imino protons from tetrads $\mathrm{G} 2$ to $\mathrm{U} 7$ remain sharp even above $80^{\circ} \mathrm{C}$.

The spectra recorded in $\mathrm{H}_{2} \mathrm{O}$ featured an unexpectedly sharp and thermally resistant resonance in the region characteristic to 2'-hydroxyl groups (see Fig. 1). At room temperature, this resonance had a line width only a few $\mathrm{Hz}$ higher than the sharpest resonances of nonexchangeable protons, indicating a very attenuated rate of exchange with the solvent. This resonance was unambiguously assigned to $2^{\prime} \mathrm{OH}$ of U7 by the observation of the cross peaks to U7-H2' in the TOCSY and COSY spectra (Supplemental Fig. S1). Apart from the NOEs to the protons of its own ribose, several contacts to the protons of the preceding residue were also observed for $\mathrm{U} 7-2^{\prime} \mathrm{OH}$ (among which the strongest were to $\mathrm{G} 6-\mathrm{H}^{\prime}$ and $\mathrm{G} 6-\mathrm{H} 8$ ).

Based on the information presented so far, one could propose a crude model of the quadruplex under study. It would feature the four strands twisting around each other following helical paths, while adapting backbone angles

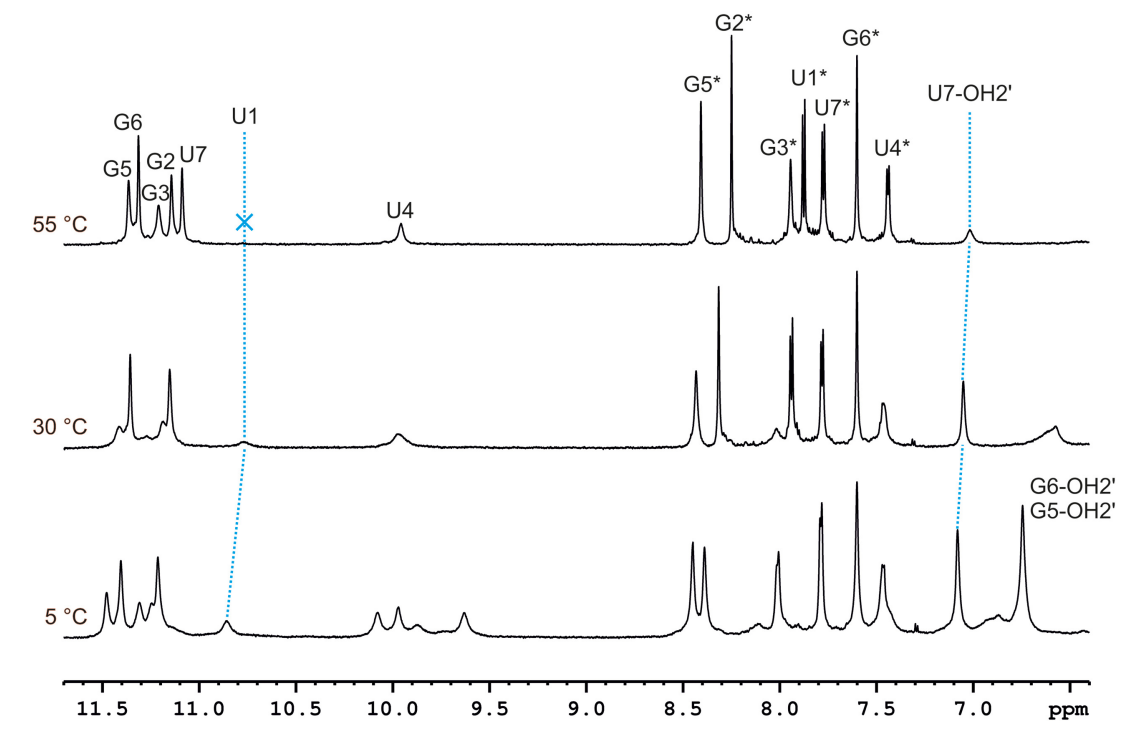

FIGURE 1. Imino and aromatic regions of the 1D NMR spectra of $r(U G G U G G U)_{4}$ in the $\mathrm{K}^{+}$-containing buffer at three different temperatures. The assignments of the imino and aromatic protons are given with residue numbers with the aromatic peaks additionally marked with asterisks (*). The U7-2' $\mathrm{OH}$ proton is also highlighted. close to A-form RNA. While the majority of the measured NOE contacts would indeed support a similar model, there remain several important NOESY cross-peaks which cannot be explained by it. They mostly include contacts between residues $\mathrm{G} 6$ and $\mathrm{U7}$, and the two most notable of those peaks-namely $\mathrm{G} 6-\mathrm{H} 3^{\prime}$ to $\mathrm{U} 7-\mathrm{H}^{\prime}$ and $\mathrm{G} 6-\mathrm{H} 8$ to $\mathrm{U} 7-\mathrm{H} 2^{\prime}$ - have volumes comparable to the $\mathrm{H} 5-\mathrm{H} 6$ crosspeaks of uridines. Together with the contacts involving the $\mathrm{U} 7-2^{\prime} \mathrm{OH}$, as well as, a series of weaker peaks, they report on an unexpectedly close spatial proximity of the U7 ribose to both the sugar and the base of G6. Such close contact may either be due to an unusual backbone conformation or alternatively be caused by formation of a higher order structure. We have investigated the possibility of quadruplex dimerization through small angle $X$-ray scattering (SAXS) measurements. Both the radius of gyration and the molecular volume estimates extracted from the SAXS profile are in excellent agreement with what would be expected for a tetramolecular quadruplex (Supplemental Table S4). Moreover, the scattering curve back-calculated for the tetrameric structure that we solve by NMR (see below) is in excellent agreement with the experimentally measured one $\left(\chi^{2}=1.04\right)$, while a model of the octamer fits the data very poorly $\left(\chi^{2}=15.49\right)$ (Supplemental Fig. S6). Overall, the SAXS data leave no doubt that the quadruplex under study exists exclusively as a tetrameric species and thus the unexpected NMR signals cannot be explained by the formation of higher order assemblies. A second group of NOEs that do not conform to the simple "A-form like" model is located in the proximity of the G2/ G3 step. Most notable among them are the unexpectedly strong $\mathrm{G} 2-\mathrm{H} 1^{\prime}$ to $\mathrm{G} 3-\mathrm{H} 8$ and $\mathrm{G} 3-\mathrm{H} 5^{\prime}$ to $\mathrm{G} 3-\mathrm{H} 8$ contacts, suggesting spatial proximity of below $3 \AA$ (both these atom pairs would be separated by $\sim 5 \AA$ in a standard backbone conformation).

The J-couplings bring further confirmation of the presence of unusual conformations in the two parts of the structure just outlined. Five out of seven sugar rings adapt a $\mathrm{C}^{\prime}$-endo conformation, as best illustrated by the lack of $\mathrm{H}^{\prime}{ }^{\prime}-\mathrm{H} 2^{\prime}$ cross peaks in the DQF-COSY and TOCSY spectra $\left({ }^{3} \mathrm{~J}_{\mathrm{H} 1^{\prime}-\mathrm{H} 2^{\prime}} \approx 0\right)$. On the other hand the $\mathrm{G} 2$ and $\mathrm{U} 7$ residues exhibit ${ }^{3} \mathrm{~J}_{\mathrm{H} 1^{\prime}-\mathrm{H} 2^{\prime}}$ around $8 \mathrm{~Hz}$ and thus appear to be locked in a C2'-endo conformation.

The phosphorus spectrum of r(UGGUGGU $)_{4}$ features a surprisingly high dispersion of chemical shift values, in the range from -5 to $-2 \mathrm{ppm}$ (Supplemental Fig. S2). Only the phosphorous atoms at the U1/G2 and U4/G5 steps resonate within the 
range of chemical shifts characteristic to the typical (gauche-/gauche-) rotamer of the $\alpha / \zeta$ angles (Gorenstein 1984), providing another hint of the presence of atypical backbone conformations. Interestingly, for the quadruplex under study the NMR spectra recorded in $\mathrm{K}^{+}$- and $\mathrm{Na}^{+}$-containing solutions are almost identical, with all the chemical shifts of nonexchangeable protons differing by $<0.15 \mathrm{ppm}$ between the two conditions (Supplemental Tables S1, S2). Also the sets of NOESY cross-peaks (including their intensities), the sugar puckers and phosphate chemical shifts measured in the two conditions are very similar, both containing all the "unusual" features outlined throughout this section. All these similarities allow us to conclude that the structures formed by $\mathrm{r}(\mathrm{UGGUGGU})$ in the presence of $\mathrm{K}^{+}$ and $\mathrm{Na}^{+}$are practically identical. The only pronounced spectral difference between these two conditions is related to the imino protons of $\mathrm{G} 3$ and $\mathrm{U} 4$. In both cases, the bulk of the imino resonances occupies a spectral window of around $0.6 \mathrm{ppm}$ (from 11.2 to 11.8 in $\mathrm{Na}^{+}$and from 10.85 to 11.5 in $\mathrm{K}^{+}$at $5^{\circ} \mathrm{C}$ ), each time with one imino proton resonating significantly upfield from that region. In the potassium buffer it is the U4 imino proton (at 9.98 ppm), while unexpectedly in the sodium conditions it is the one belonging to $\mathrm{G} 3$ (at $10.58 \mathrm{ppm}$ ). This difference is most likely related to the distinct ways in which the two types of cations organize themselves in the channel of the G-quadruplex.

\section{NMR structure of $r(U G G U G G U)_{4}$}

The NMR structure of $r(U G G U G G U)_{4}$ was solved through a restrained MD protocol, described in Materials and Methods. The structure was released in the PDB under the ID: 6GE1. A representative structure from the NMR bundle is presented in Figure 2. The overall fold of the molecule is indeed that of a four-stranded, parallel quadruplex. However, what is also apparent is an abrupt reversal of the direction of each chain near its $3^{\prime}$ terminus. Indeed, the phosphodiester backbone makes an almost $180^{\circ}$ turn between $\mathrm{G} 6$ and $\mathrm{U7}$, achieved by adapting unusual values of the $\alpha / \zeta$ dihedral angles $\alpha=139^{\circ} \pm 16^{\circ}, \zeta=50^{\circ} \pm 3^{\circ}$ (the G6/U7 suite falls within the $5 z$ category in the classification of RNA rotamers by Richardson et al. 2008). On the other hand, the central part of the quadruplex, that is suites G3/U4, U4/G5 and G5/G6, adapt backbone dihedral values akin to the standard A-form RNA. The same is also true for the $5^{\prime}$-terminal U1/G2 suite, with the exception of the presence of the $\mathrm{C} 2$ '-endo sugar pucker on $\mathrm{G} 2$. On the other hand, the backbone conformation at the G2/G3 step is highly unusual, featuring a trans-rotamer at the $\gamma$ angle as well as uncommon values of the $\alpha$ and $\zeta$ angles. Thus, the presence of unexpected NOE contacts in the 3'-terminal region and around $\mathrm{G} 3$ does translate into uncommon backbone dihedrals in these parts of the final NMR structure, while the rest of the molecule features the expected, standard rotamers.

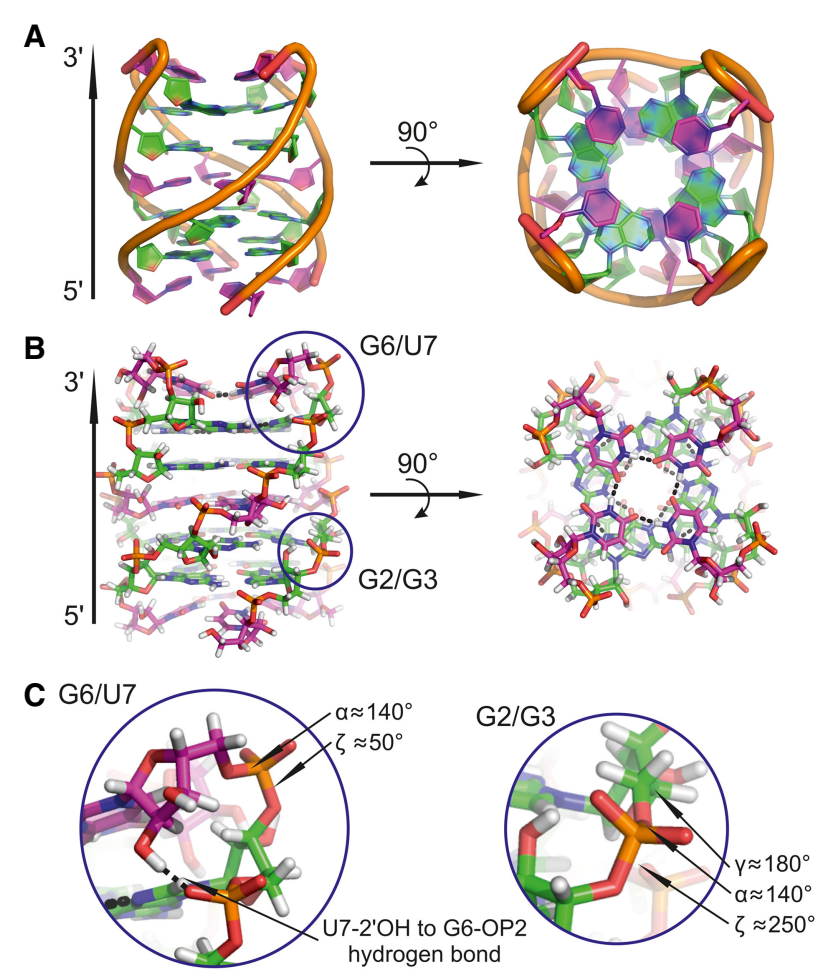

FIGURE 2. The NMR structure of r(UGGUGGU) $)_{4}$ in the $(A)$ "cartoon" and $(B)$ "all atom" representations. Panel $C$ features close-up views of some structural regions of interest, marked also on panel $B$. The G-tetrads and U-tetrads are colored green and magenta, respectively.

The backbone conformation also correlates well with the chemical shift pattern observed in the phosphorus spectra (Supplemental Fig. S2). The two most downfield shifted phosphorus resonances are indeed those belonging to the G3 and U7 phosphate groups, featuring uncommon values of the $\alpha$ and/or $\zeta$ dihedrals. Out of the four remaining phosphorus atoms, which all exist in an A-form like structural context, G2 and G5 resonate in the chemical shift range considered typical for such conformations, while $U 4$ and $G 6$ are once again somewhat downfield shifted. For these two phosphorous atoms, the origin of the shift is not clear, but it may be related to their involvement in hydrogen bonding interactions (see below).

The conformation of the $3^{\prime}$ terminus, featuring a "reversed" $U$ residue, is the most important feature of the presented structure. The backbone reversal still allows for the easy formation of the $\mathrm{U} 7 \mathrm{U}$-tetrad, yet the uridine base is forced to stack on $\mathrm{G} 6$ with the opposite face (Fig. 2). At the same time, U7 conserves the typical anti-conformation of the glycosidic torsion angle $\chi$ and, as a consequence, the direction of the hydrogen bonding within this tetrad is opposite to that found throughout the rest of the molecule (Supplemental Fig. S3). Such conformation also brings the sugar moieties of G6 and U7 into close proximity, thus explaining the observed strong NOE contacts between the protons of these two riboses. 
Importantly, the "reversed-U tetrad" motif provides a structural basis for the unusually strong solvent-exchange protection of the U7-2'OH proton. Namely, it puts the U7-2'OH group at hydrogen bonding distance from the phosphate group of G6 (Fig. 2). Indeed, the U7-2'OH proton is involved in the formation of a hydrogen bond with G6-OP2 in all of its occurrences in the final structural bundle. Unfortunately, the presence of such a hydrogen bond cannot be directly inferred from the NMR spectrum. Reassuringly, a range of indirect NMR evidence is available, supporting the $U 7-2^{\prime} \mathrm{OH}$ to G6-OP2 hydrogen bond formation. In the hydrogen-bonded conformation, the $\mathrm{U} 7-2^{\prime} \mathrm{OH}$ is pointing toward the $\mathrm{H}^{\prime}$ ' proton of its own ribose and away from the $\mathrm{H}^{\prime}{ }^{\prime}$. Such an arrangement is in perfect agreement with the observed NOE intensities, as the volume of the $\mathrm{U} 7-2^{\prime} \mathrm{OH}$ cross peak to $\mathrm{H}^{\prime}$ is always more than twice as high as that of its cross peak to $\mathrm{H}^{\prime}$. Several other NOEs (to $\mathrm{G} 6-\mathrm{H} 8, \mathrm{G} 5-\mathrm{H}_{3}$ ' and weak to $\mathrm{G} 5$ $\mathrm{H} 2^{\prime}$ ) also restrict the $\mathrm{U} 7-2^{\prime} \mathrm{OH}$ proton to point in the general direction of the $\mathrm{G} 6$ phosphate. Another source of information about the orientation of the $2^{\prime} \mathrm{OH}$ groups in RNA resides in the value of the ${ }^{3} \mathrm{~J}_{\mathrm{H}^{\prime}-2^{\prime} \mathrm{OH}}$ scalar coupling. For the $U 7$ residue, this coupling can be estimated to be very small (1-2 Hz), based on: (i) the very weak $\mathrm{H} 2^{\prime}-2^{\prime} \mathrm{OH}$ cross peak in the TOCSY spectrum and (ii) the practically unchanged line width of the $\mathrm{H} 2^{\prime}$ proton between $\mathrm{H}_{2} \mathrm{O}$ and $\mathrm{D}_{2} \mathrm{O}$ (U7-H2' lies at 3.98 ppm-outside of the envelope of the other sugar signals-and thus its line width can be evaluated directly from 1D proton spectra). In the presented structure the $\mathrm{H} 2^{\prime}-\mathrm{C}^{\prime}-\mathrm{O} 2^{\prime}-2^{\prime} \mathrm{OH}$ dihedral angle assumes the value of $59^{\circ} \pm 5^{\circ}$. According to the Karplus relation for the ${ }^{3} \mathrm{~J}_{\mathrm{H}^{\prime}-2^{\prime} \mathrm{OH}}$ coupling (Fohrer et al. 2006), such a conformation should give rise to a coupling in the $0-2 \mathrm{~Hz}$ range, in perfect agreement with what we measure. The formation of the U7-2'OH to G6-OP2 hydrogen bond may also explain a downfield shift of $\mathrm{G} 6$ phosphorus, despite it being located in an A-form structural context. Overall, despite being unable to directly point out the hydrogen bond acceptor for the $U 7-2^{\prime} \mathrm{OH}$ proton, the gathered NMR data not only contain evidence that this proton is indeed involved in strong hydrogen bonding (solvent-exchange protection even at elevated temperatures), but also provide a plethora of indirect indications (NOE intensities, J-coupling, phosphorus shift) that its hydrogen bond acceptor is indeed the G6 phosphate group.

The second novel structural feature found in the r(UGGUGGU) ${ }_{4}$ quadruplex is constituted by its central tetrad U4. The U-tetrad fits into the core of a G-quadruplex surprisingly well without inducing any nonstandard structural features in its direct proximity, even despite its somewhat smaller dimensions. Both the G3/U4 and U4/G5 steps adapt typical A-form like backbone conformations. Each U4 stacks reasonably well with G3 (partial overlap with the six-membered ring) while it has very little overlap with the base of G5. In all the lowest energy conformers, the U4 tetrad is not ideally planar, with each uridine being slightly inclined in the direction of $\mathrm{G} 5$. The reason for this inclination may be the electrostatic repulsion between the U4-O4 and G3-O6 atoms which lay just vis-à-vis each other along the helical axis of the quadruplex (while the G5-O6 atoms are located halfway between two instances of the U4 base).

The $5^{\prime}$ terminus of the quadruplex is capped with another U-tetrad, formed by the U1 residues. This tetrad adapts a bowl-like shape, with each uridine considerably inclined toward G2.

\section{MD simulations of $r(U G G U G G U)_{4}$}

In order to gain insight into the structural features not easily accessible to NMR spectroscopy, such as interactions with metal ions and solvent molecules, the system was subjected to extensive ( $6 \mu \mathrm{sec}$ in total) explicit solvent MD simulations.

\section{The number of cations in the quadruplex core}

The first series of simulations was aimed at assessing what are the most likely ways in which potassium ions organize themselves in the channel of the r(UGGUGGU) quadruplex. Three distinct simulations were thus performed, in which four, five or six potassium ions were manually placed into the quadruplex channel, prior to the start of the dynamics (the simulations UGG_4K, UGG_5K, and UGG_6K, respectively_see Table 1; Fig. 3). In each of the simulations, after initial minor structural rearrangements with respect to the NMR ensemble (see below), the system remained very stable for the rest of the dynamics. Almost no events involving the exchange of cations between the channel of the quadruplex and the bulk solution were observed. Let us now review the distinctive features of the three simulations. In the MD run UGG_4K, the ions were initially placed between two consecutive G-tetrads (G2-G3 and G5-G6), as well as between the terminal U-tetrads and their neighboring G-tetrads (U1-G2 and G6-U7), leaving the center of the channel empty (Fig. 3A). The only rearrangement of the cations in the channel occurred after just 50 nsec of dynamics, when the $\mathrm{K}^{+}$ion, initially placed between the $\mathrm{G} 5$ and $\mathrm{G} 6$ tetrads, relocated itself to a position between $U 4$ and $\mathrm{G} 5$ (Fig. 3A). The resulting configuration remained unchanged until the end of the simulation. Regarding the structure, correct geometry of the tetrads was preserved throughout the run.

In the run featuring $5 \mathrm{~K}^{+}$in the quadruplex channel (UGG_5K), the initial placement of ions was similar to that described above, with the fifth ion added in the center of the U4 tetrad (Fig. 3B). During this simulation, the four ions in the peripheries of the channel generally conserved their initial positions, while the central ion first briefly positioned itself between U4 and G5 (for around $15 \mathrm{nsec}$ ) and 
TABLE 1. Selected structural features of $r\left(\right.$ UGGUGGU) ${ }_{4}$ monitored over the course of the different MD simulations

\begin{tabular}{|c|c|c|c|c|c|c|c|}
\hline \multirow[b]{2}{*}{$\begin{array}{l}\text { Simulation } \\
\text { name }\end{array}$} & \multirow[b]{2}{*}{$\begin{array}{l}\text { Channel } \\
\text { ions } \\
\text { (initial) }\end{array}$} & \multirow[b]{2}{*}{$\begin{array}{l}\mathrm{U} 7-2^{\prime} \mathrm{OH} \text { to } \\
\text { G6-OP2 } \mathrm{H} \text {-bond } \\
\text { conservation }\end{array}$} & \multicolumn{2}{|c|}{$\begin{array}{l}\text { Hydrogen bonding } \\
\text { in the G-tetrads }\end{array}$} & \multirow[b]{2}{*}{$\begin{array}{c}\text { Occupancy of the } \\
\text { "reversed } \\
\text { U-tetrad" ion- } \\
\text { binding pocket }\end{array}$} & \multicolumn{2}{|c|}{$\begin{array}{c}\text { Presence of well-defined } \\
\text { hydration pattern around U4 }\end{array}$} \\
\hline & & & $\begin{array}{l}\text { Fraction of } \\
\text { standard } \\
\mathrm{H} \text {-bonds } \\
(\mathrm{H} 1-\mathrm{O} 6)\end{array}$ & $\begin{array}{c}\text { Fraction of } \\
\text { bifurcated } \\
\mathrm{H} \text {-bonds } \\
(\mathrm{H} 1-\mathrm{N} 7)\end{array}$ & & $\begin{array}{l}3 \mathrm{H} \text {-bonds } \\
\text { conserved } \\
\text { (see text) }\end{array}$ & $\begin{array}{l}4 \mathrm{H} \text {-bonds } \\
\text { conserved } \\
\text { (see text) }\end{array}$ \\
\hline UGG_4K & $4 \mathrm{~K}^{+}$ & $99.8 \%$ & $96.2 \%$ & $2.1 \%$ & $71.4 \%$ & $87.0 \%$ & $58.2 \%$ \\
\hline UGG_5K & $5 \mathrm{~K}^{+}$ & $99.7 \%$ & $74.4 \%$ & $25.2 \%$ & $71.4 \%$ & $90.2 \%$ & $64.8 \%$ \\
\hline UGG_6K & $6 \mathrm{~K}^{+}$ & $99.5 \%$ & $73.4 \%$ & $26.2 \%$ & $71.1 \%$ & $89.4 \%$ & $63.5 \%$ \\
\hline UGG_LiMerz & $4 \mathrm{~K}^{+}$ & $99.2 \%$ & $90.4 \%$ & $8.9 \%$ & $37.7 \%$ & $86.3 \%$ & $55.7 \%$ \\
\hline UGG_TIP3P & $4 \mathrm{~K}^{+}$ & $97.6 \%$ & $96.3 \%$ & $2.0 \%$ & $39.9 \%$ & $80.1 \%$ & $41.7 \%$ \\
\hline UGG_Na & $7 \mathrm{Na}^{+}$ & $97.1 \%$ & $59.6 \%$ & $42.6 \%$ & $0 \%$ & $82.0 \%$ & $54.4 \%$ \\
\hline
\end{tabular}

then moved between $\mathrm{G} 3$ and $\mathrm{U} 4$ for the rest of the run (Fig. 3B). Structurally, the quadruplex remained globally unchanged during the dynamics; however, the geometries of the G-tetrads were often distorted by the formation of bifurcated hydrogen bonds (Fig. 3B), quantitative analysis of hydrogen bonding modes in all three simulations can be found in Table 1.

The third simulation, UGG_6K, started with a fully occupied channel (Fig. 3C). However, after around $70 \mathrm{nsec}$ of dynamics the quadruplex expelled the metal ion closest to its $3^{\prime}$ terminus from the channel. This event actually involved a concerted motion of three channel ions (Fig. 3C) toward the 3'-terminal exit of the channel and thus lead to the formation of a vacancy between U4 and G5. Moreover, this simulation featured the most severe distortions of the hydrogen bonding geometries of the tetrads, with the $\mathrm{G} 3$ tetrad almost instantly assuming a conformation stabilized by only a single hydrogen bond ( $\mathrm{H} 1-\mathrm{N} 7)$. After the number of channel ions is reduced to five, the geometry of the G3 tetrad evolves into an arrangement featuring bifurcated hydrogen bonds, as observed in the UGG_5K simulation.

Thus, to summarize the differences in the tetrad geometry between the three simulations: The quadruplex harboring $4 \mathrm{~K}^{+}$ions in its channel remained the most true to the ideal geometries of the G-tetrads, the one containing $5 \mathrm{~K}^{+}$was plagued with the widespread appearance of bifurcated hydrogen bonds, while the molecule loaded with $6 \mathrm{~K}^{+}$was even forced to transiently adapt a completely different mode of hydrogen bonding in one of its tetrads. Thus, in our simulations the optimal number of potassium ions in the central channel of $r(U G G U G G U)_{4}$ appears to be four, located at the U1/G2, G2/G3, U4/G5, and G6/U7 steps, respectively (Fig. 3A). However, one should interpret this result with caution, as it is known that the AMBER description where ions have assigned point char- ges can lead to overestimation of the inter-ion repulsive forces (Gkionis et al. 2014; Islam et al. 2015). Also, the tendency to form bifurcated hydrogen bonds is a known artifact in MD simulations of G-quadruplexes (Špačková et al. 1999; Havrila et al. 2017). Keeping these reservations in mind, we will nevertheless adapt UGG_4K as our reference simulation for the rest of the paper.

For completeness, we also note that some minor structural changes with respect to the NMR ensemble, unrelated to the number of channel cations, were present in all of the simulations. First, in the unrestrained dynamics the ribose of $\mathrm{U} 1$ is in rapid exchange between $\mathrm{N}$ - and S-conformations (see Supplemental Fig. S4), while the NMR data clearly indicate a pure $\mathrm{N}$-type pucker. Second, significant changes of both $\alpha$ and $\beta$ dihedral angles occur at the G2/G3 step in the unrestrained MD (see Supplemental Fig. S4). These changes seem to compensate each other to a large extent and thus their impact on the relative positions of the $\mathrm{G} 2$ and $\mathrm{G} 3$ residues is rather limited. Finally, similar $\varepsilon / \zeta$ transitions can be also observed at the G6/U7 step, yet, only in a minor population of structures, rapidly exchanging with "NMR-like" conformations. The first of the observed changes clearly conflicts with the experimental data. In turn, the other two represent transitions to conformations favored by the dihedral terms of the applied force field. We are thus strongly inclined to interpret these changes as simulation artifacts. Anyway, due to their local character, these rearrangements have a very minor impact on the overall fold of the quadruplex.

\section{The conformation of the U7-2' $\mathrm{OH}$ group}

A very important feature, recurrent in all the simulations, is the exceptionally high stability of the $\mathrm{U} 7-2^{\prime} \mathrm{OH}$ to G6-OP2 hydrogen bonding throughout the dynamics. In each run the said hydrogen bond was present for over $99 \%$ of the 
A

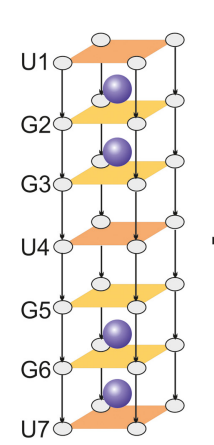

\section{UGG_4K}
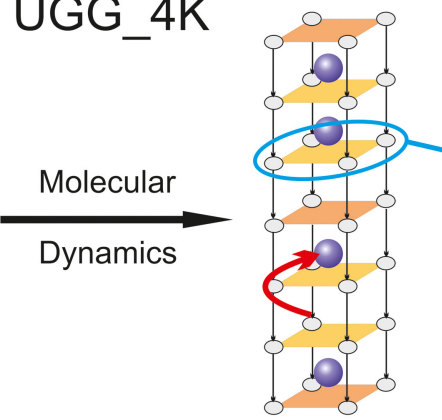

B

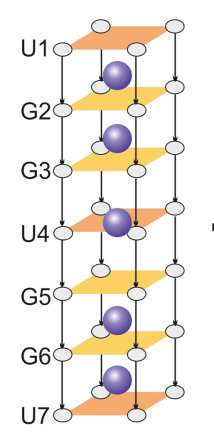

C
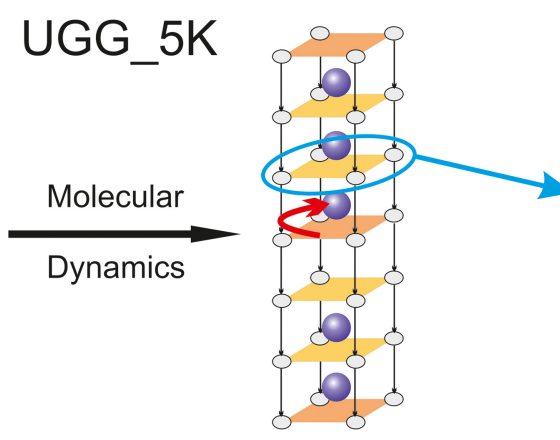

\section{UGG_6K}

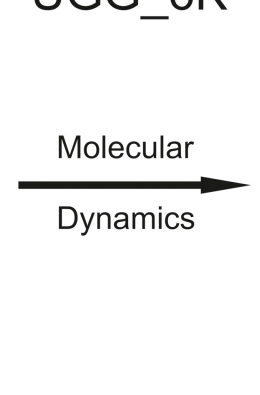

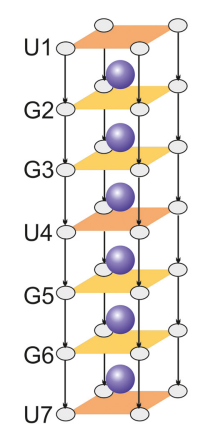

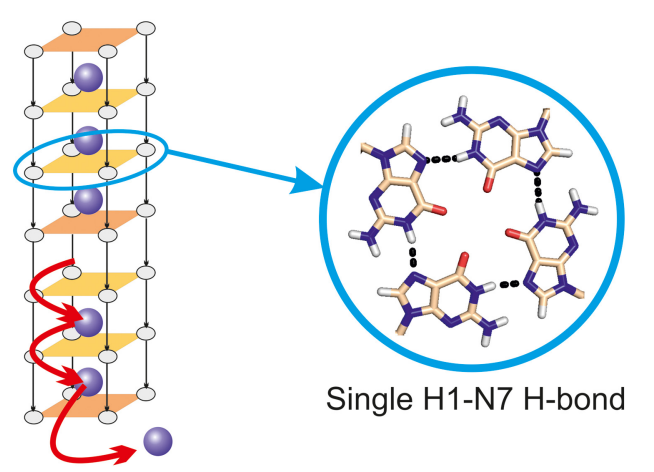

FIGURE 3. The effect of the number on $\mathrm{K}^{+}$ions in the quadruplex channel on the simulation behavior of r(UGGUGGU) 4 . Each panel presents the initial (left) and final (center) positioning of the channel ions, as well as their impact on the tetrad geometries sampled during the simulations (right). The simulations presented were run with $4(A), 5(B)$, or $6(C) \mathrm{K}^{+}$ions in the channel. do-density map" of potassium ions and water molecules around the simulated quadruplex, visualizing the positions in space in which the ions and solvent molecules were found notoriously often during the UGG_4K simulation. Such an analysis reveals the presence of four additional potassium ions, bound in the groove between G6 and U7. The position of each cation is very well defined and the potassium occupancy at each site is above $70 \%$ (Table 1). Cations bound in these new "reversed-U" binding sites are partially dehydrated, forming specific interactions with U7-O2' and U7-O2 atoms of one strand and $\mathrm{G} 6-\mathrm{O} 2^{\prime}$ and G6-N3 of the neighboring one (all the $\mathrm{O}-\mathrm{K}$ and $\mathrm{N}-\mathrm{K}$ distances around $3 \AA$; Fig. 4). In contrast to the potassium bound inside the channel, the ions interacting in the new site are more labile, with average residence time of the order of $1 \mathrm{nsec}$. To ascertain that the observed ion binding was not just an artifact of the way in which potassium was described in the simulation, two more $1 \mu \mathrm{sec} M D$ runs were performed. The first of them-UGG_LiMerz-made use of an alternative parametrization of potassium ions (Li et al. 2015). In the second simulation (UGG_TIP3P), the TIP3P model was used for the water molecules instead of SPC/E. Both new simulations confirmed the binding of $\mathrm{K}^{+}$cations in the loop formed by the reversed uridine. Also, the mode of binding was unaffected by the changes in the MD force field. On time (as evaluated using simple geometric distance/angle criteria described in Materials and Methods), which was even higher than the degree of preservation of some of the hydrogen bonds in the tetrads. The unrestrained MD simulations thus strongly advocate for the very stable U7$2^{\prime} \mathrm{OH}$ to G6-OP2 hydrogen bonding, further reinforcing the conclusions from NMR.

\section{Specific potassium binding pocket in the vicinity of the reversed $U$-tetrad}

The most unexpected and thought-provoking feature present in all of the performed simulations is the formation of additional, specific potassium binding sites in the "reversed" 3 '-terminal region. Figure 4 depicts the "pseu- the other hand, the choice of cation/solvent parameters seems to have some effect on the apparent affinity of $\mathrm{K}^{+}$ ions, as both the new simulations feature a noticeably diminished occupancy of these sites (to around 40\%; Table 1).

\section{Stable hydration pattern around U4}

The quadruplex grooves are highly hydrated throughout the dynamics, yet most water molecules are interacting with them very transiently. However, our simulations also reveal three specific, well-defined positions along the groove that are consistently occupied by the water molecules over the course of the dynamics. Two of them are located in the central part of the quadruplex on the level of the U4 tetrad. When bound, these water molecules are 


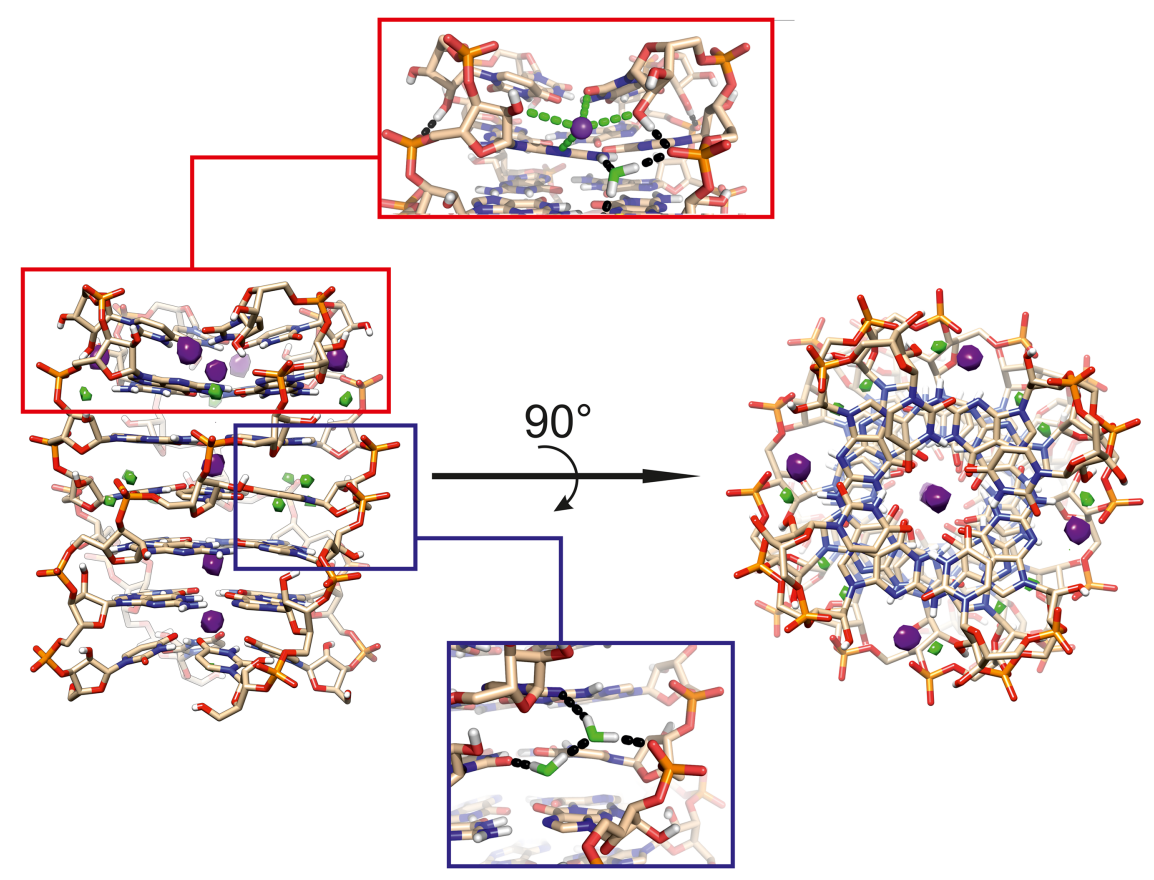

FIGURE 4. Specific interactions of $r(U G G U G G U)_{4}$ with $\mathrm{K}^{+}$ions and water molecules observed in the UGG_4K simulation. The central panel depicts positions repeatedly adapted by $\mathrm{K}^{+}$ions (purple) and water oxygen atoms (green) around the quadruplex (i.e., $\mathrm{K}^{+}$and water "density maps"). The insets present the atomic details of each quadruplex/ion and quadruplex/solvent interaction (with hydrogen bonds depicted as black dashes and coordination bonds as green dashes). by $\mathrm{Na}^{+}$cations. In brief, all the small structural rearrangements encountered in the $\mathrm{K}^{+}$simulations were once again present and also a significant population of tetrads with bifurcated hydrogen bonding was observed. The simulation in $\mathrm{Na}^{+}$have also preserved the U7-OH2' to G6OP2 hydrogen bond just as well as the previous runs and displayed a similar hydration pattern (Table 1). On the other hand, the most striking difference was the complete lack of interaction of $\mathrm{Na}^{+}$ions with the new "reversed- $U$ " binding site, established in the $\mathrm{K}^{+}$-containing simulations. In the currently discussed run, this site was for the part of time occupied by a water molecule, hydrogen bonding to G6-O2' and U7-O2', but not a single instance of a sodium ion entering it was observed. The lack of affinity of sodium ions toward the new site could arise from their higher hydration energy as compared to $\mathrm{K}^{+}$, because ion binding to the discussed site requires partial dehydration. involved in an intricate network of hydrogen bonds U4$\mathrm{O} 2 . . . \mathrm{HOH} \ldots \mathrm{O}(\mathrm{H}) \mathrm{H} . . . \cup 4-\mathrm{OP} 2$ depicted in Figure 4. This hydrogen bonding pattern is present in as many as $87 \%$ of frames in the UGG_4K simulation. In around two-thirds of these frames the hydrogen bonding network is further augmented by an additional interaction between the second water molecule and the G5-N3 atom. The third specifically binding water molecule bridges the phosphate groups of $\mathrm{G} 5$ and $\mathrm{G} 6$, while its oxygen atom is also interacting with one of the amino protons of $\mathrm{G} 6$ (with around $60 \%$ occupancy). Importantly, the described hydration patterns repeat themselves in all the performed runs (Table 1). The formation of such a stable solvent-solute hydrogen bond network may be an important factor stabilizing the $U$-tetrad formed inside the $\mathrm{G}$-quadruplex stem (first two bound waters).

\section{MD simulation in the presence of $\mathrm{Na}^{+}$}

Our experimental data show that the structure of $r(U G G U G G U)_{4}$ is practically the same in $\mathrm{K}^{+}$and $\mathrm{Na}^{+}$-containing solutions. In order to check whether the modes of ion-quadruplex interactions are also preserved, we performed a $1 \mu \mathrm{sec}$ simulation utilizing $\mathrm{Na}^{+}$as the counterion. As $\mathrm{Na}^{+}$ions are smaller than $\mathrm{K}^{+}$and also more mobile in MD simulations of G-quadruplexes, we have started this simulation with the quadruplex channel fully occupied

\section{DISCUSSION}

\section{The conformation of the $3^{\prime}$-terminal uridine-the reversed U-tetrad}

Previous studies have reported a surprisingly high stabilizing effect of 3 '-terminal U-tetrads in tetramolecular RNA Gquadruplexes (Cheong and Moore 1992; Xu et al. 2010; Zhou et al. 2017), which the available structural picture of this motif fails to explain. The solution structure of r(UGGUGGU) $)_{4}$ brings new important insights which help to bridge this gap. First of all, our NMR data show that the 3'-terminal U-tetrad adapts a rigid, well-defined conformation in solution. The stability of the NMR determined conformation on the $\mu \mathrm{sec}$ timescales is further confirmed by the performed MD simulations. The picture of a rigid arrangement of the $3^{\prime}$ terminal U-tetrad, emerging from our data, is to some extent at odds with what was earlier observed in the crystal structures of similar motifs (Fyfe et al. 2015). On the one hand, a conformation practically identical to the arrangement we observe in solution was also the one most often encountered in the crystals. For example, the structures deposited under PDBIDs: 4XKO (Chen et al. 2015), 4RNE (Fyfe et al. 2015), or 2GRB (Pan et al. 2006b) contain uniquely this kind of arrangement. On the other hand, alternative arrangements of the $3^{\prime}$-terminal U-tetrad were also present (e.g., PDBID: 1J8G [Deng 
et al. 2001], 1J6S [Pan et al. 2003a], 4RKV [Fyfe et al. 2015] and 4RJ1 [Fyfe et al. 2015]), sometimes in the same crystals as the "reversed" conformation. Thus, overall the crystalline data indicated the presence of three distinct and possibly interconverting conformations of the $3^{\prime}$-terminal Utetrad. Yet, one has to take into account that the $3^{\prime} \mathrm{U}$-tetrad represents a peripheral structural element, whose environment differs significantly between the solution and crystalline states. In the crystal, the quadruplex molecules most often stack on each other forming quasi-infinite columns, usually with the coordination of an additional metal ion between the quadruplexes. In other words, the structural diversity present for the $3^{\prime}$-terminal U-tetrads in some Xray structures of similar quadruplexes may constitute an artifact of the crystal packing. This apparent structural diversity may have been the reason why not much attention was ever attributed to the "reversed U-tetrad" conformation. Our data establish the presence of the "reversed U-tetrad" motif in RNA G-quadruplexes in solution as a rigid structural element.

Even more importantly, our data also bring forward some intrinsic features of this motif, likely responsible for making it so energetically favorable. First and foremost, we observe the presence of a very stable hydrogen bonding interaction between the U7-2' $\mathrm{OH}$ group and the phosphate group of the preceding guanosine G6. Also the MD simulations strongly suggest that this hydrogen bonding is preserved practically all of the time. The NMR observation that the $\mathrm{U} 7-2^{\prime} \mathrm{OH}$ resonance line width has only a minor contribution from solvent exchange around room temperature constitutes an important proof of the structural rigidity of the reversed U-tetrad motif. Structurally, bringing the $\mathrm{U} 7-2^{\prime} \mathrm{OH}$ group and the G6-OP2 atom into hydrogen bonding distance requires not only the abrupt turn of the backbone between $\mathrm{G} 6$ and U7, but also the presence of C2'-endo pucker in U7. This explains why we observe uniquely the $\mathrm{C} 2^{\prime}$-endo pucker for U7, both in the NMR experiment and throughout the MD simulations. Interestingly, a set of backbone dihedral angles practically identical to the one we observe at the G6/U7 step, was found previously in the dinucleotide step forming the first turn of the so-called "S-motif", present in the sarcin-ricin loop of rRNA (Correll et al. 1998, 2003). The literature data on the S-motif also report the 2'-OH to phosphate hydrogen bonding at this dinucleotide step, as a factor stabilizing this structural element (Correll et al. 1998, 2003; Richardson et al. 2008). In our case, a considerably stronger stabilizing effect should be expected, as the hydrogen bonding interaction is repeated in each of the four strands.

The second factor which can also significantly contribute to the stabilization of the "reversed U-tetrad" is its interaction with potassium ions. This specific potassium binding, observed in the MD simulations, occurs in the quadruplex groove in the region of the G6/U7 step and up to four electron-pair donating groups from the quadruplex appear to be directly involved in the interaction (U7-O2', U7-O2, G6O2', G6-N3). This means that the potassium ion interacts with this site with partial dehydration and thus its binding can potentially stabilize the system both enthalpically (favorable electrostatic interactions) and entropically (release of water molecules). Our current results, however, do not provide direct insights into the energetics of this interaction. Strikingly, the MD simulations performed in $\mathrm{Na}^{+}$-containing conditions show no interaction of this ion with the newly established binding pocket. This effect may be related to the significantly higher hydration energy of $\mathrm{Na}^{+}$, as compared to $\mathrm{K}^{+}$. The lack of $\mathrm{Na}^{+}$binding near the "reversed U-tetrad" may also partially explain our previous observation that the r(UGGUGGU) ${ }_{4}$ quadruplex is significantly less thermally stable in the presence of $\mathrm{Na}^{+}$than in the $\mathrm{K}^{+}$-containing buffer (Malgowska et al. 2014).

\section{The formation of a U-tetrad within a G-stem}

The presented structure also contains the first observation of a U-tetrad stacked between two G-tetrads. Up to now uridine residues embedded in such a structural context were observed to adapt bulged out conformations rather than U-tetrads (crystal structures 1P79 [Pan et al. 2003c] and 2AWE [Pan et al. 2006a]). The only reported occurrences of U-tetrads inside quadruplex stems involved very unusual contexts related to the dimerization of tetramolecular quadruplexes, in which at least one of the neighboring tetrads was not a G-tetrad (2AWE; Pan et al. 2006a; Xiao et al. 2017). In our structure of r(UGGUGGU) ${ }_{4}$, the central $\cup 4$ tetrad appears to fit well into the G-stem, without inducing excessive over- or under-twisting of the helices. Despite its apparently seamless integration into the quadruplex core, the $\mathrm{U} 4$ tetrad still differs from its $\mathrm{G}$-counterparts in at least two aspects: (i) it has somewhat smaller dimensions and (ii) it exposes into the quadruplex groove the $\mathrm{O} 2$ atoms which may act as hydrogen bond acceptors, while a G-tetrad exposes amine groups capable of being hydrogen bond donors instead. The results of our MD simulations strongly suggest that the different properties of the U-tetrad are enough to affect the number of potassium ions that can be accommodated inside the quadruplex channel. Not a single time throughout our simulations has the system spontaneously adapted a conformation featuring a simultaneous presence of potassium ions on both sides of the central U-tetrad. Moreover, when such a configuration was imposed on the system by manual placement of ions in the vicinity of U4 (simulation UGG_6K), it led to a severe distortion of the geometry of one of the neighboring G-tetrads. One can thus conclude that the presence of a U-tetrad inside a G-quadruplex stem diminishes-by at least one-the number of potassium ions that can be hosted by the quadruplex channel. This result is in line with our previous MS measurements, which showed that r(UGGUGGU) $)_{4}$ exists 
predominantly in forms bound to 4 or 5 ions (Malgowska et al. 2014). The second specific feature of the central Utetrad-the presence of the hydrogen bond acceptor atom $\mathrm{O} 2$ in the quadruplex groove-is most likely the driving force behind the formation of a very stable hydration pattern in this part of the groove. In all of our MD simulations a double water bridge connecting the U4-O2 atom of one strand and U4-OP2 of the neighboring one is recurring with very high populations (>80\%). The presence of such a well-conserved network of interactions may constitute an important factor favoring the U-tetrad formation over bulging out of the uridine residues.

One should also mention that our NMR data report on the presence of an exchange process in the direct vicinity of the central tetrad, affecting several protons belonging to $\mathrm{G} 3$ and $U 4$ residues. The motions are in intermediate regime at room temperature and accelerate to fast exchange at $55^{\circ} \mathrm{C}$. The collected NMR and MD data do not allow us to draw definitive conclusions on the nature of these motions. The possible motional processes involved may include ion movement in the channel across the U4 tetrad or some backbone rearrangements at the G2/G3 dinucleotide step featuring an unusual rotamer in the dominating conformer.

\section{The $5^{\prime}$-terminal U-tetrad}

The $5^{\prime}$-terminal uridine residue in $r(U G G U G G U)_{4}$ is also involved in the formation of a U-tetrad. However, in contrast with the other two U-tetrads, the $5^{\prime}$-terminal one is not particularly thermally stable, with its imino proton disappearing from the NMR spectrum somewhat above room temperature. This lower thermal stability is consistent with the observations made in a previous work reporting a quadruplex fold containing a $5^{\prime}$-terminal U-tetrad (Cheong and Moore 1992).

The comparison of the two structures reveals another interesting common feature. Namely, these two solution structures display the exact same unusual set of backbone dihedrals at the G2/G3 step. Interestingly, when the same quadruplex $r(U G G G G U)_{4}$ is crystallized (PDBID: 1J8G) and the $U 1$ residue is bulged out due to dimerization, the $G 2$ / G3 step adapts the typical A-form. Thus, all this indirect evidence appear to connect the formation of the $5^{\prime}$-terminal U-tetrad with the appearance of unusual backbone conformation at the neighboring GG step.

\section{Conclusions}

We studied the properties of U-tetrads in three very different structural contexts: on the $3^{\prime}$ and $5^{\prime}$ termini of a tetramolecular RNA quadruplex and in the middle of its stem, stacked between two G-tetrads. Our results demonstrate that the 3'-terminal U-tetrad, contrary to the previous observations (Fyfe et al. 2015), adapts a single, well-defined con- formation. This conformation involves an abrupt turn of the backbone and leads to an inversion of the hydrogen bond directionality in the $3^{\prime}$-terminal U-tetrad. The exceptional thermal stability of G-quadruplexes containing a 3'-terminal U-tetrad is well-documented (Cheong and Moore 1992; Xu et al. 2010; Zhou et al. 2017). The conformation we observe contains some important features which can constitute the structural basic for this effect. Namely, we demonstrate that the "reversed U-tetrad" motif enables the formation of four additional very stable U7-OH2' to G6-OP2 hydrogen bonds, as well as, we bring forward the possibility of specific potassium binding in the pocket formed by the backbone turn. We postulate the "reversed U-tetrad" to be the general mode of 3 '-capping occurring in tetramolecular quadruplexes with $3^{\prime}$-terminal uridine residues, which endows this family of structures with an increased thermal stability through the mechanisms just outlined. The formation of tetramolecular RNA G-quadruplexes with 3'-terminal U-tetrads has not been, as for now, observed in vivo. Nevertheless, short oligonucleotides derived from the human telomeric repeat were demonstrated to form such structures in vitro (Xu et al. 2010; Xiao et al. 2017). The remarkable thermal stability of these folds has inspired the authors of these studies to postulate that such structures are indeed very likely to be assumed also in the in vivo conditions.

The studied quadruplex also contains another U-tetrad embedded inside a regular G-stem-a structural feature that was never observed before. We note that the reduced dimensions of a U-tetrad do not lead to marked structural distortions within the stem, yet its presence decreases the amount of ions that can be accommodated by the quadruplex channel. We also observe a stable hydration pattern in the quadruplex groove vis-à-vis this tetrad, a factor likely contributing to the preference for the U-tetrad formation over bulging the uridine out of the stem. Finally, the $5^{\prime}$-terminal uridine residue also participates in U-tetrad formation, unlike what is usually observed in crystal structures.

Overall, our findings underline the capability of uridine residues in RNA G-quadruplexes to form tetrads in a variety of structural contexts. Moreover, they argue that it is exactly the structural context that dictates the geometry and the properties of the formed tetrad, as well as the impact that it has on the quadruplex as a whole. This context dependent behavior of U-tetrads may constitute a factor bringing more diversity into the otherwise rather structurally homogeneous RNA G-quadruplexes.

\section{MATERIALS AND METHODS}

\section{RNA sample preparation}

The r(UGGUGGU) oligoribonucleotide was obtained using standard phosphoramidite chemistry. Similarly to our previous work (Malgowska et al. 2014), the oligoribonucleotide was synthetized 
with an additional $5^{\prime}$-phosphate group in order to improve the sample homogeneity (prevent quadruplex dimerization).

The purified oligoribonucleotide was divided into two parts. One was dissolved in $50 \mathrm{mM} \mathrm{KCl}, 10 \mathrm{mM} \mathrm{K} \mathrm{HPO}_{4} / \mathrm{KH}_{2} \mathrm{PO}_{4}$, $0.1 \mathrm{mM}$ EDTA, pH 6.8 (potassium buffer) while the second part was dissolved in $150 \mathrm{mM} \mathrm{NaCl}, 10 \mathrm{mM} \mathrm{Na} 2 \mathrm{HPO}_{4} / \mathrm{NaH}_{2} \mathrm{PO}_{4}$, $0.1 \mathrm{mM}$ EDTA, pH 6.8 (sodium buffer). Both samples were annealed by heating at $90^{\circ} \mathrm{C}$ for $5 \mathrm{~min}$ and then slowly cooled down to the room temperature and stored at $4^{\circ} \mathrm{C}$. The measurements were done after $3 \mathrm{wk}$ in order to allow the formation of the tetramolecular G-quadruplexes.

\section{NMR spectroscopy}

All NMR spectra were collected using a $700 \mathrm{MHz}$ Bruker Avance III spectrometer equipped with a $\mathrm{QCl}$ CryoProbe. The resonance assignment of nonexchangeable protons was achieved through a combined analysis of sets of NOESY, TOCSY, HC-HSQC, HPCOSY, and DQF-COSY spectra recorded in $100 \% \mathrm{D}_{2} \mathrm{O}$ at $25^{\circ} \mathrm{C}$. In turn, the exchangeable proton resonances were assigned using NOESY spectra measured in $90 \% \mathrm{H}_{2} \mathrm{O} / 10 \% \mathrm{D}_{2} \mathrm{O}$ at $5^{\circ} \mathrm{C}$ and $25^{\circ} \mathrm{C}$. The aromatic portion of the assigned NOESY spectrum is presented in Supplemental Figure S5. For the sample dissolved in potassium buffer, an additional set of spectra was recorded at $55^{\circ} \mathrm{C}$. All the spectra were processed using the Topspin software (version 3.5) and then analyzed in NMRFAM-Sparky (Lee et al. 2015).

\section{NMR structure calculations}

The distance restraints between nonexchangeable protons were extracted from the peak volumes in NOESY spectra recorded in $\mathrm{D}_{2} \mathrm{O}$ with 100 and 150 ms mixing times. The H5-H6 cross-peaks of uridine residues and ribose $\mathrm{H}^{\prime}-\mathrm{H} 5^{\prime \prime}$ cross-peaks were used for distance calibration. The remaining NOESY cross-peaks were

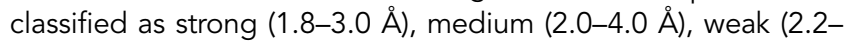
$5.0 \AA)$, and very weak (2.4-6.0 A). The distance restraints between exchangeable protons were derived from the NOESY spectra recorded in $\mathrm{H}_{2} \mathrm{O}$ (150 ms mixing) using a similar procedure. The mean volume of the imino-amino contacts of guanosines was used as an approximate ruler and all other cross-peaks were classified into three groups: strong (1.8-3.0 A), medium (2.5-4.5 $)$, and weak (3.0-6.5 $\AA$ ). The analysis of the ${ }^{3} \mathrm{~J}_{\mathrm{HH}}$ couplings allowed to fix the conformation of the ribose to $\mathrm{C}^{\prime}$-endo for U1, G3, U4, G5, and $\mathrm{G} 6$ and to $C 2$ '-endo for $\mathrm{G} 2$ and U7. Moreover, for several residues $(\mathrm{G} 2, \mathrm{U} 4, \mathrm{G} 5, \mathrm{G} 6, \mathrm{U} 7)$ the $\mathrm{P}-\mathrm{H} 4$ ' cross peaks were observed which allowed us to restrain the $\beta$ and $\gamma$ dihedral angles for these residues to $180^{\circ}$ and $60^{\circ}$, respectively $\left( \pm 60^{\circ}\right)$. As the conformation of all the ribose rings was fixed using J-coupling data, no intra-ribose NOEs were used in the structure determination, except those involving $\mathrm{H}^{\prime} / \mathrm{H}^{\prime \prime}$ protons. Overall, a total of 160 NOE, 10 backbone torsion angle and 7 sugar pucker restraints were obtained (per strand).

The structure calculation procedure consisted of two major steps: (i) initial folding from random extended conformations, performed in Xplor-NIH (Schwieters et al. 2003) and (ii) refinement of the pre-folded structures using AMBER16 (Case et al. 2017). The first step involved a simulated annealing procedure in the dihedral angle space (Schwieters and Clore 2001). The system was first evolved at $3000 \mathrm{~K}$ for $15 \mathrm{psec}$ and then cooled to $25 \mathrm{~K}$ over the course of the next $150 \mathrm{psec}$, and finally minimized in the Cartesian space. As only a single set of resonances was present in the NMR spectra, each observed NOESY peak may correspond to either an intra- or inter-strand interaction and thus ambiguous restraints were used (with the SUM averaging option). To enforce the formation of the base tetrads, hydrogen bond restraints were applied. As the available NMR data do not indicate which specific strand is the acceptor of each such hydrogen bond (or in other words does not specify the directionality of each tetrad) these restraints were also treated as ambiguous. A total of 1000 structures was calculated and the 20 best of them were taken as input for the refinement procedure.

The structure was refined by simulated annealing in the Cartesian space in explicit solvent using AMBER16. Each of the Xplor structures was solvated in a truncated octahedral box of SPC/E water and neutralized by addition of potassium ions. The specifics of the force field used are described in the "Unrestrained Molecular Dynamics" section. In the preparation phase, the solvated system was first heated under constant volume to $300 \mathrm{~K}$ with harmonic restraints on the solute over the course of 100 psec and then equilibrated at this temperature under constant pressure for another $100 \mathrm{psec}$ (still with harmonic restraints). At this step the harmonic restraints were removed and substituted by the experimental NMR restraints. The system was kept at $300 \mathrm{~K}$ for another $25 \mathrm{psec}$ and then gradually cooled to $0 \mathrm{~K}$ over the next 75 psec (at a constant box volume). No planarity or symmetry restraints were enforced during the AMBER simulations.

A total of 500 conformers was calculated and the 15 best of them were selected to form the final NMR bundle, based on fidelity to both experimental data and ideal covalent geometries. The final bundle of structures is very well defined, with a pairwise RMSD of $0.68 \AA$, and contains no violations of the NOE (>0.5 $)$ ), dihedral or hydrogen bonding restraints (Supplemental Table S3). Atomic coordinates and the list of experimental restraints for the reported NMR structure have been deposited with the protein data bank under accession number 6GE1, while the chemical shifts have been deposited at the BMRB under the number 34265 .

\section{SAXS data collection and analysis}

SAXS experiments were performed at beamline P12 of Petra III storage ring. Twenty microliters of the sample and the corresponding matching buffer were loaded into a 96-well plate. Data collection was performed at $10^{\circ} \mathrm{C}$. Integration, scaling and buffer subtraction were accomplished using the program Primus (Konarev et al. 2003). SAXS data were collected over the s range of 0.0088-4 $\mathrm{nm}^{-1}$. Overlays of the merged data sets were used to detect concentration dependent scattering in the lowest $s$ region. Indirect Fourier transform of the SAXS scattering curve was performed with Gnom (Svergun 1992). The SAXS profile with the lowest noise and free of interparticle interference was subsequently used for ab initio modeling with the program DAMMIN. For each refined and merged SAXS curve, multiple independent DAMMIN runs were performed, superimposed, and averaged with the program DAMAVER (Volkov and Svergun 2003). Theoretical calculation of radii of gyration and SAXS curves were performed using CRYSOL (Svergun et al. 1995). The details of SAXS data collection and analysis are gathered in Supplemental Table S4, while Supplemental Figure S6 presents the fitting of the SAXS curve by the NMR structure. 


\section{Unrestrained molecular dynamics}

All the simulations were performed using the parm $99 \mathrm{bsc} 0 \chi_{\mathrm{OL} 3}$ force field (Cheatham et al. 1999; Pérez et al. 2007; Zgarbová et al. 2011), which as for AMBER 16 is the recommended force field for the simulations of RNA. Unless stated otherwise, the SPC/E water model and Joung-Cheatham parameters for monovalent cations were used (Joung and Cheatham 2008). After the appropriate number of $\mathrm{K}^{+} / \mathrm{Na}^{+}$cations was manually placed in the central channel of the first conformer of the NMR bundle, the system was solvated in a truncated octahedral box of water and neutralized. Finally, additional $\mathrm{K}^{+} / \mathrm{Na}^{+}$and $\mathrm{Cl}^{-}$ ions were added to obtain $0.2 \mathrm{M}$ salt concentration (on top of the cations used for neutralization). The preparation stage of each performed run consisted of the following steps: (i) minimization with harmonic restraints on the solute and channel cations, (ii) minimization without harmonic restraints, (iii) heating to $300 \mathrm{~K}$ (at constant volume) over the course of $100 \mathrm{psec}$ with harmonic restraints on the solute and channel cations, (iv) pressure equilibration (in NPT ensemble) over the course of 100 psec with harmonic restraints on the solute and channel cations, (v) 2 nsec of MD (in NPT system) restrained with the experimental NMR data, and (vi) another 2 nsec of restrained MD during which the weight of NMR restraints was gradually reduced to 0 .

During the following $1 \mu \mathrm{sec}$ production runs, the integration step size was set to $2 \mathrm{fsec}$ (with SHAKE algorithm [Ryckaert et al. 1977] in use), the particle mesh Ewald method (Darden et al. 1993; Essmann et al. 1995) was used to treat the electrostatic potential terms with direct space nonbonded cutoff $9 \AA$ and the temperature was controlled with the Langevin thermostat. The NPT (ntb =2) simulation was carried out at $300 \mathrm{~K}$ and $1 \mathrm{~atm}$ with a Berendsen barostat, default compressibility of the system and pressure relaxation time taup $=1.0 \mathrm{psec}$. Coordinate information was collected every 5 psec.

\section{Analysis of MD trajectories}

All the AMBER trajectories were analyzed using CPPTRAJ (Roe and Cheatham 2013) and a series of in-house Python scripts. The $\mathrm{K}^{+}$and water density maps around the average MD structure (Fig. 4) were calculated using the CPPTRAJ "grid" routine and visualized using UCSF-Chimera (Pettersen et al. 2004). For the identification of hydrogen bonds (Table 1) the standard CPPTRAJ routine was used, with a $3.4 \AA$ distance cutoff between heavy atoms and $\mathrm{a} 135^{\circ}$ angle cutoff for the $\mathrm{X}-\mathrm{H}$........ Y atom triple. The $\mathrm{K}^{+}$ binding in the G6/U7 turn region was quantified by assessing the presence (or lack thereof) of a $\mathrm{K}^{+}$ion in the $1.5 \AA$ radius from the geometric center of the [G6-O2' (one strand), U7-O2' (neighboring strand)] atom selection (in the idealized bound conformation-see Figure 4 - the $\mathrm{K}^{+}$ion is located approximately half way between these two ligand atoms). All the results presented in Table 1 ( $\mathrm{H}$-bonds, $\mathrm{K}^{+}$binding) were averaged over the four independent strands of the quadruplex.

\section{SUPPLEMENTAL MATERIAL}

Supplemental material is available for this article.

\section{ACKNOWLEDGMENTS}

This work was supported by the National Science Center (Poland) under grant agreements UMO-2014/13/B/ST5/04144 to Z.G. and UMO-2013/08/A/ST5/00295 to R.K. This publication was also supported by the Polish Ministry of Science and Higher Education under the KNOW program. The calculations were performed at Poznań Supercomputing and Networking Center.

Received July 20, 2018; accepted October 16, 2018.

\section{REFERENCES}

Agarwala P, Pandey S, Maiti S. 2015. The tale of RNA G-quadruplex. Org Biomol Chem 13: 5570-5585. doi:10.1039/C4OB02681K

Arora A, Maiti S. 2009. Differential biophysical behavior of human telomeric RNA and DNA quadruplex. J Phys Chem B 113: 10515-10520. doi:10.1021/jp810638n

Azzalin CM, Reichenbach P, Khoriauli L, Giulotto E, Lingner J. 2007. Telomeric repeat containing RNA and RNA surveillance factors at mammalian chromosome ends. Science 318: 798-801. doi:10.1126/science.1147182

Biffi G, Di Antonio M, Tannahill D, Balasubramanian S. 2014. Visualization and selective chemical targeting of RNA G-quadruplex structures in the cytoplasm of human cells. Nat Chem 6: 75-80. doi:10.1038/nchem.1805

Cáceres C, Wright G, Gouyette C, Parkinson G, Subirana JA. 2004. A thymine tetrad in d(TGGGGT) quadruplexes stabilized with $\mathrm{Tl}^{+} / \mathrm{Na}^{+}$ ions. Nucleic Acids Res 32: 1097-1102. doi:10.1093/nar/gkh269

Case DA, Cerutti DS, Cheatham TEI, Darden TA, Duke RE, Giese TJ, Gohlke H, Goetz AW, Greene D, Homeyer N, et al. 2017. AMBER 2017. University of California, San Francisco, CA.

Cheatham TE III, Cieplak P, Kollman PA. 1999. A modified version of the Cornell et al. force field with improved sugar pucker phases and helical repeat. J Biomol Struct Dyn 16: 845-862. doi:10.1080/07391102.1999.10508297

Chen MC, Murat $P$, Abecassis K, Ferré-D'Amaré AR, Balasubramanian S. 2015. Insights into the mechanism of a G-quadruplex-unwinding DEAH-box helicase. Nucleic Acids Res 43: 2223-2231. doi:10.1093/nar/gkv051

Cheong C, Moore PB. 1992. Solution structure of an unusually stable RNA tetraplex containing G- and U-quartet structures. Biochemistry 31: 8406-8414. doi:10.1021/bi00151a003

Correll CC, Munishkin A, Chan YL, Ren Z, Wool IG, Steitz TA. 1998. Crystal structure of the ribosomal RNA domain essential for binding elongation factors. Proc Natl Acad Sci 95: 13436-13441. doi:10.1073/pnas.95.23.13436

Correll CC, Beneken J, Plantinga MJ, Lubbers M, Chan Y. 2003. The common and the distinctive features of the bulged-G motif based on a $1.04 \AA$ resolution RNA structure. Nucleic Acids Res 31: 6806-6818. doi:10.1093/nar/gkg908

Creze C, Rinaldi B, Haser R, Bouvet P, Gouet P. 2007. Structure of a $\mathrm{d}\left(\mathrm{TGGGGT)}\right.$ quadruplex crystallized in the presence of $\mathrm{Li}^{+}$ions. Acta Crystallogr D Biol Crystallogr 63: 682-688. doi:10.1107/ S0907444907013315

Darden T, York D, Pedersen L. 1993. Particle mesh Ewald: an N.log(N) method for Ewald sums in large systems. J Chem Phys 98: 1008910092. doi:10.1063/1.464397

Deng J, Xiong Y, Sundaralingam M. 2001. X-ray analysis of an RNA tetraplex (UGGGGU) ${ }_{4}$ with divalent $\mathrm{Sr}^{2+}$ ions at subatomic resolution (0.61 Å). Proc Natl Acad Sci 98: 13665-13670. doi:10.1073/ pnas. 241374798

Didiot MC, Tian Z, Schaeffer C, Subramanian M, Mandel JL, Moine H. 2008. The G-quartet containing FMRP binding site in FMR1 mRNA 
is a potent exonic splicing enhancer. Nucleic Acids Res 36: 4902-4912. doi:10.1093/nar/gkn472

Essmann U, Perera L, Berkowitz ML, Darden T, Lee H, Pedersen LG. 1995. A smooth particle mesh Ewald method. J Chem Phys 103: 8577-8593. doi:10.1063/1.470117

Fohrer J, Hennig M, Carlomagno T. 2006. Influence of the 2'-hydroxyl group conformation on the stability of A-form helices in RNA. $J$ Mol Biol 356: 280-287. doi:10.1016/j.jmb.2005.11.043

Fyfe AC, Dunten PW, Martick MM, Scott WG. 2015. Structural variations and solvent structure of r(UGGGGU) quadruplexes stabilized by $\mathrm{Sr}^{2+}$ ions. J Mol Biol 427: 2205-2219. doi:10.1016/j. jmb.2015.03.022

Gkionis K, Kruse H, Platts JA, Mládek A, Koča J, Šponer J. 2014. Ion binding to quadruplex DNA stems. Comparison of MM and QM descriptions reveals sizable polarization effects not included in contemporary simulations. J Chem Theory Comput 10: 13261340. doi:10.1021/ct4009969

Gorenstein DG. 1984. Phosphorus-31 chemical shifts: principles and empirical observations. In Phosphorous-31 NMR (ed. Gorenstein DG), pp. 7-36. Academic Press, NY.

Gu J, Leszczynski J. 2001. A theoretical study of thymine and uracil tetrads: structures, properties, and interactions with the monovalent $\mathrm{K}^{+}$cation. J Phys Chem A 105: 10366-10371. doi:10.1021/ jp004550v

Havrila M, Stadlbauer P, Islam B, Otyepka M, Šponer J. 2017. Effect of monovalent ion parameters on molecular dynamics simulations of G-quadruplexes. J Chem Theory Comput 13: 3911-3926. doi:10.1021/acs.jctc.7b00257

Islam B, Stadlbauer P, Krepl M, Koca J, Neidle S, Haider S, Sponer J. 2015. Extended molecular dynamics of a c-kit promoter quadruplex. Nucleic Acids Res 43: 8673-8693. doi:10.1093/nar/gkv785

Joachimi A, Benz A, Hartig JS. 2009. A comparison of DNA and RNA quadruplex structures and stabilities. Bioorg Med Chem 17: 6811-6815. doi:10.1016/j.bmc.2009.08.043

Joung IS, Cheatham TE III. 2008. Determination of alkali and halide monovalent ion parameters for use in explicitly solvated biomolecular simulations. J Phys Chem B 112: 9020-9041. doi:10.1021/ jp8001614

Konarev PV, Volkov WV, Sokolova AV, Koch MHJ, Svergun DI. 2003. PRIMUS: a windows PC-based system for small-angle scattering data analysis. J Appl Crystallogr 36: 1277-1282. doi:10.1107/ S0021889803012779

Laughlan G, Murchie Al, Norman DG, Moore MH, Moody PC, Lilley DM, Luisi B. 1994. The high-resolution crystal structure of a parallel-stranded guanine tetraplex. Science 265: 520-524. doi:10.1126/science.8036494

Lee MPH, Parkinson GN, Hazel P, Neidle S. 2007. Observation of the coexistence of sodium and calcium ions in a DNA G-quadruplex ion channel. J Am Chem Soc 129: 10106-10107. doi:10.1021/ ja0740869

Lee W, Tonelli M, Markley JL. 2015. NMRFAM-SPARKY: enhanced software for biomolecular NMR spectroscopy. Bioinformatics 31: 1325-1327. doi:10.1093/bioinformatics/btu830

Li P, Song LF, Merz KM. 2015. Systematic parameterization of monovalent ions employing the nonbonded model. J Chem Theory Comput 11: 1645-1657. doi:10.1021/ct500918t

Lyons SM, Gudanis D, Coyne SM, Gdaniec Z, Ivanov P. 2017. Identification of functional tetramolecular RNA G-quadruplexes derived from transfer RNAs. Nat Commun 8: 1127. doi:10.1038/ s41467-017-01278-w

Malgowska M, Gudanis D, Kierzek R, Wyszko E, Gabelica V, Gdaniec Z. 2014. Distinctive structural motifs of RNA G-quadruplexes composed of AGG, CGG and UGG trinucleotide repeats. Nucleic Acids Res 42: 10196-10207. doi:10.1093/nar/gku710
Malgowska M, Czajczynska K, Gudanis D, Tworak A, Gdaniec Z. 2016. Overview of the RNA G-quadruplex structures. Acta Biochim Pol 63: 609-621. doi:10.18388/abp.2016_1335

Marcel V, Tran PL, Sagne C, Martel-Planche G, Vaslin L, TeuladeFichou MP, Hall J, Mergny JL, Hainaut P, Van Dyck E. 2011. Gquadruplex structures in TP53 intron 3: role in alternative splicing and in production of p53 mRNA isoforms. Carcinogenesis 32: 271-278. doi:10.1093/carcin/bgq253

Mergny JL, De Cian A, Ghelab A, Saccà B, Lacroix L. 2005. Kinetics of tetramolecular quadruplexes. Nucleic Acids Res 33: 81-94. doi: 10.1093/nar/gki148

Millevoi S, Moine H, Vagner S. 2012. G-quadruplexes in RNA biology. Wiley Interdiscip Rev RNA 3: 495-507. doi:10.1002/wrna.1113

Olsen CM, Marky LA. 2009. Energetic and hydration contributions of the removal of methyl groups from thymine to form uracil in Gquadruplexes. J Phys Chem B 113: 9-11. doi:10.1021/jp808526d

Pan B, Xiong Y, Shi K, Deng J, Sundaralingam M. 2003a. Crystal structure of an RNA purine-rich tetraplex containing adenine tetrads: implications for specific binding in RNA tetraplexes. Structure 11: 815-823. doi:10.1016/S0969-2126(03)00107-2

Pan B, Xiong Y, Shi K, Sundaralingam M. 2003b. An eight-stranded helical fragment in RNA crystal structure: implications for tetraplex interaction. Structure 11: 825-831. doi:10.1016/S0969-2126(03) 00108-4

Pan B, Xiong Y, Shi K, Sundaralingam M. 2003c. Crystal structure of a bulged RNA tetraplex at 1.1 Å resolution: implications for a novel binding site in RNA tetraplex. Structure 11: 1423-1430. doi: 10.1016/j.str.2003.09.017

Pan B, Shi K, Sundaralingam M. 2006a. Base-tetrad swapping results in dimerization of RNA quadruplexes: implications for formation of the i-motif RNA octaplex. Proc Natl Acad Sci 103: 3130-3134. doi: 10.1073/pnas.0507730103

Pan B, Shi K, Sundaralingam M. 2006b. Crystal structure of an RNA quadruplex containing inosine tetrad: implications for the roles of $\mathrm{NH}_{2}$ group in purine tetrads. J Mol Biol 363: 451-459. doi: 10.1016/j.jmb.2006.08.022

Patel P, Hosur RV. 1999. NMR observation of T-tetrads in a parallel stranded DNA quadruplex formed by Saccharomyces cerevisiae telomere repeats. Nucleic Acids Res 27: 2457-2464. doi: 10.1093/nar/27.12.2457

Pérez A, Marchán I, Svozil D, Sponer J, Cheatham TE III, Laughton CA, Orozco M. 2007. Refinement of the AMBER force field for nucleic acids: improving the description of $\alpha / \gamma$ conformers. Biophys J 92: 3817-3829. doi:10.1529/biophysj.106.097782

Pettersen EF, Goddard TD, Huang CC, Couch GS, Greenblatt DM, Meng EC, Ferrin TE. 2004. UCSF chimera-a visualization system for exploratory research and analysis. J Comput Chem 25: 1605-1612. doi:10.1002/jcc.20084

Phillips K, Dauter Z, Murchie AlH, Lilley DMJ, Luisi B. 1997. The crystal structure of a parallel-stranded guanine tetraplex at $0.95 \AA$ resolution. J Mol Biol 273: 171-182. doi:10.1006/jmbi.1997.1292

Richardson JS, Schneider B, Murray LW, Kapral GJ, Immormino RM, Headd JJ, Richardson DC, Ham D, Hershkovits E, Williams LD, et al. 2008. RNA backbone: consensus all-angle conformers and modular string nomenclature (an RNA Ontology Consortium contribution). RNA 14: 465-481. doi:10.1261/rna.657708

Roe DR, Cheatham TE III. 2013. PTRAJ and CPPTRAJ: software for processing and analysis of molecular dynamics trajectory data. J Chem Theory Comput 9: 3084-3095. doi:10.1021/ ct400341p

Ryckaert JP, Ciccotti G, Berendsen HJ. 1977. Numerical integration of the Cartesian equations of motion of a system with constraints: molecular dynamics of n-alkanes. J Comput Phys 23: 327-341. doi:10.1016/0021-9991(77)90098-5 
Schoeftner S, Blasco MA. 2007. Developmentally regulated transcription of mammalian telomeres by DNA-dependent RNA polymerase II. Nat Cell Biol 10: 228. doi:10.1038/ncb1685

Schoeftner S, Blasco MA. 2009. A "higher order" of telomere regulation: telomere heterochromatin and telomeric RNAs. EMBO J 28: 2323-2336. doi:10.1038/emboj.2009.197

Schwieters CD, Clore GM. 2001. Internal coordinates for molecular dynamics and minimization in structure determination and refinement. J Magn Reson 152: 288-302. doi:10.1006/jmre.2001.2413

Schwieters CD, Kuszewski JJ, Tjandra N, Clore GM. 2003. The XplorNIH NMR molecular structure determination package. J Magn Reson 160: 65-73. doi:10.1016/S1090-7807(02)00014-9

Song J, Perreault JP, Topisirovic I, Richard S. 2016. RNA G-quadruplexes and their potential regulatory roles in translation. Translation (Austin) 4: e1244031.

Špačková N, Berger I, Šponer J. 1999. Nanosecond molecular dynamics simulations of parallel and antiparallel guanine quadruplex DNA molecules. J Am Chem Soc 121: 5519-5534. doi:10.1021/ ja984449s

Svergun DI. 1992. Determination of the regularization parameter in indirect-transform methods using perceptual criteria. J Appl Crystallogr 25: 495-503. doi:10.1107/S0021889892001663

Svergun D, Barberato C, Koch MHJ. 1995. CRYSOL-a program to evaluate X-ray solution scattering of biological macromolecules from atomic coordinates. J Appl Crystallogr 28: 768-773. doi: $10.1107 /$ S0021889895007047
Varani G, Aboul-ela F, Allain FHT. 1996. NMR investigation of RNA structure. Prog Nucl Magn Reson Spectrosc 29: 51-127. doi: 10.1016/0079-6565(96)01028-X

Volkov VV, Svergun DI. 2003. Uniqueness of ab initio shape determination in small-angle scattering. J Appl Crystallogr 36: 860-864. doi:10.1107/S0021889803000268

Wijmenga SS, van Buuren BNM. 1998. The use of NMR methods for conformational studies of nucleic acids. Prog Nucl Magn Reson Spectrosc 32: 287-387. doi:10.1016/S0079-6565(97)00023-X

Xiao CD, Ishizuka T, Zhu XQ, Li Y, Sugiyama H, Xu Y. 2017. Unusual topological RNA architecture with an eight-stranded helical fragment containing A-, G-, and U-tetrads. J Am Chem Soc 139: 2565-2568. doi:10.1021/jacs.6b12274

Xu Y, Ishizuka T, Kimura T, Komiyama M. 2010. A U-tetrad stabilizes human telomeric RNA G-quadruplex structure. J Am Chem Soc 132: 7231-7233. doi:10.1021/ja909708a

Zgarbová M, Otyepka $M$, Šponer J, Mládek A, Banáš $P$, Cheatham TE III, Jurečka P. 2011. Refinement of the Cornell et al. nucleic acids force field based on reference quantum chemical calculations of glycosidic torsion profiles. J Chem Theory Comput 7: 2886-2902. doi:10.1021/ct200162x

Zhou J, Amrane S, Rosu F, Salgado GF, Bian Y, Tateishi-Karimata H, Largy E, Korkut DN, Bourdoncle A, Miyoshi D, et al. 2017. Unexpected position-dependent effects of ribose G-quartets in G-quadruplexes. J Am Chem Soc 139: 7768-7779. doi:10. 1021/jacs.7b00648 

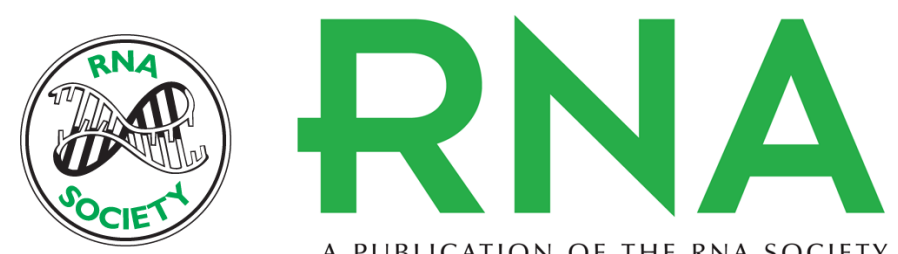

A PUBLICATION OF THE RNA SOCIETY

\section{Unraveling the structural basis for the exceptional stability of RNA G-quadruplexes capped by a uridine tetrad at the 3 ' terminus}

Witold Andralojc, Magdalena Malgowska, Joanna Sarzynska, et al.

RNA 2019 25: 121-134 originally published online October 19, 2018

Access the most recent version at doi:10.1261/rna.068163.118

\section{Supplemental http://rnajournal.cshlp.org/content/suppl/2018/10/19/rna.068163.118.DC1 \\ Material}

References This article cites 62 articles, 6 of which can be accessed free at: http://rnajournal.cshlp.org/content/25/1/121.full.html\#ref-list-1

Creative This article is distributed exclusively by the RNA Society for the first 12 months after the Commons

License full-issue publication date (see http://rnajournal.cshlp.org/site/misc/terms.xhtml). After 12 months, it is available under a Creative Commons License (Attribution-NonCommercial 4.0 International), as described at http://creativecommons.org/licenses/by-nc/4.0/.

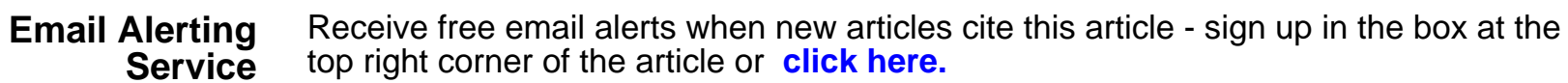

